\title{
Multicast Routing with Minimum Energy Cost and Minimum Delay in Wireless Sensor Networks"
}

\author{
Zhao Li, Wei Zhang, Hengchang Liu, Baohua Zhao, and Yugui Qu \\ Department of Computer Science, University of Science and Technology of China, \\ Laboratory of Computer Science Institute of Software Chinese Academy of Sciences, \\ Hefei, Anhui 230027, China \\ \{leezhao, bho, hcliu\}@ustc.edu, \{bhzhao, ygqu\}@ustc.edu.cn
}

\begin{abstract}
In this paper, we consider the multicast routing with minimum energy cost and minimum delay (MEMD) in wireless sensor networks under the access control scheme of Spatial Time Division Multiple Access (STDMA). We formulate and explore both MEMD multicast and ME multicast, and show that the latter is just the Maximum Leaf Spanning Tree (MLST) problem, which is NP-complete. A 2-approximation algorithm is proposed for the MLST problem through improving a known one. Based on this algorithm, an approximation algorithm is obtained for the MEMD multicast problem. To further improve the delay result, we provide another approximation algorithm for our main problem using a different approach. These algorithms are all near linear in the size of the network graph, and also are shown to have good performance by simulation results.
\end{abstract}

Keywords: Wireless Sensor Networks, STDMA, MEMD multicast, MLST.

\section{Instruction}

In typical applications of wireless sensor networks [1], battery-powered sensors are scattered throughout a monitored area, left unattended and expected to operate for a long time. Therefore, routing protocols must be optimized for ultra-low power operation in such networks [2]. Moreover, protocol design is always constrained by the following limitations of wireless sensors [3]: limited battery energy, limited transceiver resources, limited frequency resources, limited processing capability and limited storage space. For wireless sensor networks, the Time Division Multiple Access (TDMA) can be used as the access control scheme, in which the transmission capacity is divided into time slots, and each direct link is assigned a dedicated slot. A promising approach for increasing its efficiency is the STDMA scheme [4], which takes into account that sensors are usually spread out geographically, and hence sensors with a sufficient spatial separation can use the same time slot for transmission. We propose the latter as the access control scheme in our concern.

* This paper is supported by the National Natural Science Foundation of China under Grant No. 60241004, the National Grand Fundamental Research 973 Program of China under Grant No. 2003CB314801, and the State Key Laboratory of Networking and Switching Technology. 
In wireless sensor networks, multicast routing, which refers to the transmission of the same information to several destinations, is receiving an increasing interest due to its foundational applications, such as data dissemination and control information delivery. In this paper, we study the problem of multicast routing with minimum energy cost and minimum delay. Given a wireless sensor network and a multicast request, our concern is to find a multicast scheme such that the total energy cost and delay of the multicast is minimized. To our best survey, there are still no routing schemes proposed for the multicast considering these two objects together.

The contribution of our work is three-fold: Firstly, we formulate and explore the MEMD multicast problem and the ME multicast problem, and show the latter is just the MLST problem which is NP-complete. Secondly, we propose an improved 2-approximation algorithm for the MLST problem, based on a known one. Finally, we present two approximation algorithms for the MEMD multicast problem. These algorithms are all near linear in the size of the network graph and are shown to have good performance by both theoretical analysis and simulation results.

The rest of this paper is organized as follows: Section 2 posts out the related work about multicast routing and the MLST problem. We formulate and explore the MEMD multicast problem and the ME multicast problem in section 3. In Section 4, we present an approximation algorithm for the MLST problem and two other different ones for the MEMD multicast problem. Section 5 shows simulation results. We conclude this paper in Section 6.

\section{Related Work}

In recent years, many multicast routing schemes [3], [5-9] have been proposed for Ad Hoc Networks and wireless sensor networks, but none of them has considered minimum energy cost and minimum delay together. The directed diffusion scheme [8] and the two-tier data dissemination (TTDD) scheme [9] naturally support data multicasting, but they are not efficient. To improve the efficiency, the tree-based multicasting scheme can be used. In this scheme, the source and the destinations form a tree rooted at source, and the source pushes data to the destinations along the branches. In [6], Jia and Li et al discussed the energy efficient tree-based multicast problem in ad hoc wireless networks. They proved the problem is NP-hard and gave three heuristic algorithms. However, they didn't concern the multicast delay.

Given a connected undirected graph $G$, the MLST problem is to find in $G$ a spanning tree with maximum number of leaves. Using reduction from the dominating set problem Garey and Johnson have shown in [10] that this problem is NP-complete, thereafter Galbiati et al established its MAX-SNP completeness [11]. Thus there exists some constant $\varepsilon>0$ such that there is no $(1+\varepsilon)$-approximation algorithm for MLST unless $P=N P$ [12], [13]. In [14], Lu and Ravi, using a novel approach based on a notion of leafy trees, obtained a 3-approximation algorithm which works in near linear time. Solia-Oba, in [15], presented a linear 2-approximation algorithm for MLST. This algorithm first constructs a leafy forest [14] $F$ with certain expansion rules and then connects the trees of $F$ and all vertices not in $F$ to form a spanning tree of the graph $G$. To our best survey, this algorithm is the best one for this problem. The full 
algorithm is shown in the Appendix. Base on this algorithm, we propose a more efficient one in Section 4.

\section{Mathematical Programming Formulations}

Energy cost minimizing and resource allocation in STDMA [16] both are challenging problems in multicast routing design. In this section, we define and formulate the MEMD multicast problem. To further study the multicast problems, we also consider the objectives respectively and explore the ME multicast problem.

\subsection{Assumptions}

We consider a wireless sensor network $G$ with nodes set $V$, where each node of $V$ represents a stationary sensor node. STDMA is proposed as the access control scheme. Following the assumptions in [17], a direct communication link can be established between two nodes if their corresponding signal-to-noise ratio (SNR) is not lower than a certain threshold. There are several constraints and restrictions when assigning time slots. Firstly, a node can transmit or receive, but not both, in a time slot. Secondly, a node can receive data from at most one node at a time. Finally, a link is error-free only if the signal-to-interference ratio (SIR) is not lower than a threshold.

Without loss of generality, we make simplified assumptions as follows.

- All nodes broadcast multicast information at the same longest transmission radius $R$.

- A direct communication link between two nodes is established if their Euclidean distance is shorter than $R$.

- Two nodes can transmit in the same time slot without interference if the intersection of their neighbor sets is empty.

- The source node, which may be a cluster leader, a gateway or a region agent, knows the whole network topology.

- The energy cost of multicast routing can be measured only by their transmission energy since other energy cost such as receiving cost and idle cost can be simply assumed to be the same for all multicast routings.

- The multicast delay, which is the interval between the time the source nodes broadcasts the information and the time the last destination node receives it, can be measured by the total number of time slots.

Consequently, the multicast problem is to generate a spanning tree of $G$, rooted at the source node. In practice, the root generates the multicast tree and the assignment of time slots, and promulgates these schedules to all other nodes. Apparently, the time slot of a node must be behind the one of its parent node in the multicast tree. In the process of multicast routing, the root is the sponsor and all other branch nodes broadcast received multicast information at radius $R$ in their own time slots.

\subsection{Formulation for MEMD Multicast}

Under the assumptions in the section above, every branch node of the multicast tree has to broadcast the multicast information. So the multicast energy cost can be measured by 
the total number of branch nodes. Further more, each branch node should be assigned one time slot under the constraints mentioned above, and hence the total number of time slots can be used to measure the multicast delay. Consequently, the MEMD multicast problem is to generate a spanning tree of the network graph with minimum branch nodes and minimum total number of time slots. However, these two objects have no direct relation and can not be obtained at the same time. To further explore the MEMD multicast problem, we formulate it in a combinatorial optimization model in this section.

Since the multicast is tree-based, we first present a set of formulations for multicast tree (MCT), which is a spanning tree of the network. We introduce the following variables.

$$
\begin{gathered}
\operatorname{Link}((u, v))=\left\{\begin{array}{cc}
1 & \text { if }(u, v) \text { is an in link to } u \text { in the result graph } \\
-1 & \text { if }(u, v) \text { is an out link to } u \text { in the result graph } \\
0 & \text { otherwise: }(u, v) \text { is not a link in the result graph }
\end{array}\right. \\
\text { ConnectRoot }(u)= \begin{cases}1 & \text { if } u \text { is connected with the root in the result graph } \\
0 & \text { otherwise }\end{cases}
\end{gathered}
$$

The formulations set for multicast tree is as follows.

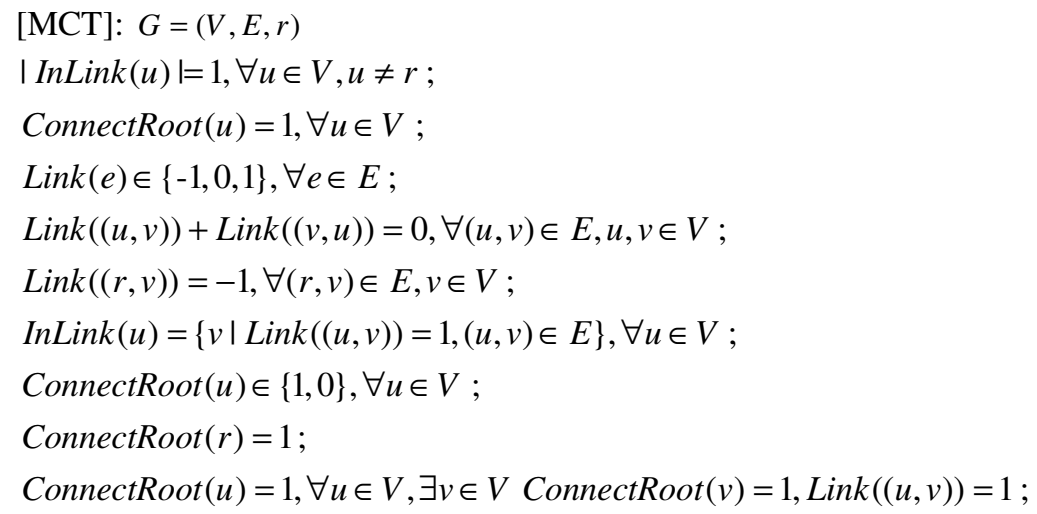

In formulations set MCT, $G$ is a connected undirected graph, and both $(u, v)$ and $(v, u)$ express the same edge between nodes $u$ and $v$. InLink $(u)$ denotes the set of all the parent nodes of node $u$. Constraint (1) restricts that the result graph has no circle and constraint (2) requires all nodes of $G$ to be connected with the root. Due to (1) and (2), the result graph must be a spanning tree of $G$. Constraint (9) presents the connection rule that a node is connected with the root only when its parent is connected with the root formerly. Other constraints define the variables and initialize them.

The formulation for MEMD multicast is shown in Formulation I. TS $(u)$ denotes the time slot assigned to node $u, \operatorname{Zone}(u)$ expresses the set of nodes that is in the transmission region of $u$, BranchNodes denotes the set of all branch nodes, and other terms retain the same meaning in the above formulations. 
Formulation I: MEMD Multicast $G=(V, E, r)$

Object: $\operatorname{Min}\left(\alpha \mid\right.$ BranchNodes $\left.\mid+\beta \operatorname{Max}_{u \in \text { BranchNodes }}(T S(u))\right), \alpha, \beta \in[0,1], \alpha+\beta=1$

Subject to:

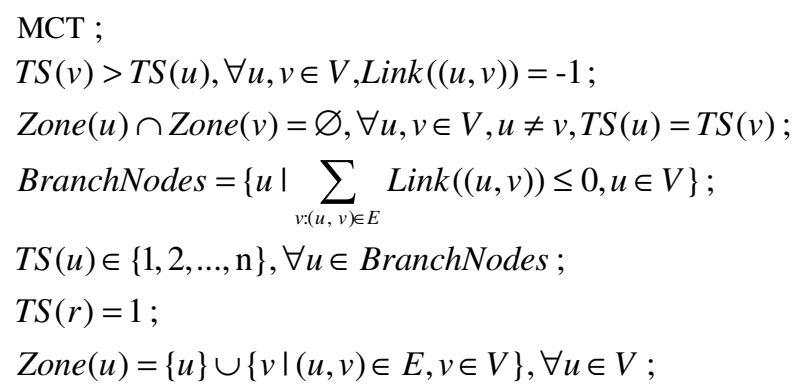

In Formulation I, the objective function (10) jointly minimizes the multicast energy cost and delay, where $\operatorname{Max}_{u \in \text { BranchNodes }}(T S(u)$ ) expresses the maximum time slot equaling to the total number of time slots in best schedules, while $\alpha$ and $\beta$ are application-specific coefficients. Constraints (11), the multicast tree formulations, ensure that the result graph of Formulation $I$ is a spanning tree of the network. Constraints (12) and (13) express the constraints of assigning time slots mentioned in the section above. Constraint (12) restricts that the time slot of a node must be behind the one of its parent node and constraint (13) ensure that nodes assigned the same time slot do not cause transmission interference. Other constraints define the variables and initialize them.

From Formulation I, we notice the MEMD multicast is a complicated problem and that it needs exponential running time to get a complete solution. To further explore the MEMD multicast, we will formulate and study the ME multicast problem in the next section.

\subsection{Formulation for ME Multicast}

Actually, considering only one object of minimum energy cost, the multicast problem is to find a spanning tree with minimum branch nodes, which is just the MLST problem. Formulation II formulates this problem, and all symbols and constraints follow Formulation I.

Formulation II: ME Multicast $G=(V, E, r)$

Object: Min | BranchNodes |

Subject to:

$$
\begin{aligned}
& \text { MCT; } \\
& \text { BranchNodes }=\left\{u \mid \sum_{v:(u, v) \in E} \operatorname{Link}((u, v)) \leq 0, u \in V\right\} ;
\end{aligned}
$$

Actually, this problem is NP-complete as introduced in Section 2. Fortunately, there are already many efficient approximation algorithms. In the following section, we 
propose an improved 2-approximation algorithm for this problem outperforming known ones.

Intuitively, the MEMD multicast problem is NP-complete or NP-hard because the MLST problem is a special case of it (when $\alpha=1$ and $\beta=0$ ). Actually, the MEMD multicast problem is even too hard to use some novel approaches such as column generation for optimal or near-optimal solutions. In the following sections, we attempt to develop two approximation algorithms for the MEMD multicast problem in different approaches.

\section{Approximation Algorithms}

In this section, we first propose a improved 2-approximation algorithm for the MLST problem based on a known one, and then present two approximation algorithms for the MEMD multicast problem using different approaches.

\subsection{An Improved 2-Approximation Algorithm for MLST}

Based on the algorithm of [15], we propose an improved 2-approximation algorithm, Imp2AppMLST $(G)$ (see Fig. 1), for the MLST problem. Like [15], this algorithm first constructs a leafy forest with certain expansion rules and then connects the trees of the forest and all vertices not in the forest to form a spanning tree of the original graph. We introduce remained degree to express the expansion rules of [15]. A vertex's remained

\begin{tabular}{|l} 
Algorithm Imp2AppMLST(G) \\
1. $\quad F:=\varnothing ;$ \\
2. $\quad$ while there is a vertex of remained degree $(R D)$ at least 3 do \\
3. $\quad$ Select a vertex $v$ of the largest remained degree; \\
4. $\quad$ Build a tree $T_{i}$ with root $v$ and expand the neighbors of $v$ as leaves; \\
5. $\quad$ while there is at least one leaf $u$ of $T_{i}$ satisfying: \\
$\quad R D(u) \geq 2$ OR $(R D(u)=1$ AND $R D$ (the only child of $u) \geq 2)$ do \\
6. $\quad$ Select from $T_{i}$ a leaf with the largest remained degree and expand it; \\
7. $\quad$ Update all vertices' remained degree information; \\
8. $\quad$ end while; \\
9. $\quad F:=F \cup T_{i} ;$ \\
10. \\
end while;
\end{tabular}

Fig. 1. An improved 2-approximation algorithm for MLST 
degree is the number of its connections that point to unexpanded vertices. The expanding vertices are sorted more elaborately by their remained degree than [15], where there are only two priorities: priority 1 and priority 2 for expanding vertices. Moreover, the trees are constructed orderly by their remained degree too.

In line 6 of $\operatorname{Imp} 2 A p p M L S T(G)$, two expanding vertices are sorted by their only child's remained degree if they have the same remained degree 1 . The while of line 5 continues if there is at least one leaf of $T_{i}$ satisfying that its remained degree is greater than 1 or its remained degree is 1 but the remained degree of its only child is greater than 1 . Due to this constraint, the result forest is leafy. Lines 5 and 6 also accord with the expansion rules of [15].

This algorithm is more efficient than the original one because it sorts the expanding vertices more elaborately. The tree generated by this algorithm is at least a 2-approximation MLST of the graph $G$ since this algorithm is a special case of the original one. The running time $\Theta\left(n^{2}+m\right)$ required by this algorithm is almost linear in the size of $G$ (nodes number and edges number), where $n$ is the number of nodes and $m$ is the number of edges.

\subsection{Algorithm I for MEMD Multicast}

In this section, we present Algorithm I (see Fig. 2) for the MEMD multicast problem. This algorithm first calls the algorithm $\operatorname{Imp2AppMLST(G)}$ to obtain a 2-approximation MLST of the network graph and then assigns time slots to all branch nodes based on the tree.

Algorithm I assigns time slots to all branch nodes round by round, spreading from the root. $A S$ denotes the set of vertices that can be assigned time slots in a round and

\section{Algorithm I $G=(V, E, r)$}

1. Call $\operatorname{Imp} 2 \operatorname{App} M L S T(G)$ to obtain a spanning tree $T$ of $G$;

2. $A S:=\{r\}$;

3. $T S:=0$;

4. while $A S \neq \varnothing$ do

5. Find a maximum subset $S$ of $A S$ randomly satisfying: the vertices of $S$ can be assigned the same time slot without transmission interference;

6. Assign each vertex of $S$ time slot $T S+1$;

7. $T S++$;

8. Update $A S$ : put the children of each vertex of $S$ in $A S$;

9. end while;

Fig. 2. Algorithm I for MEMD Multicast 
should be updated round by round, while $S$ denotes the vertices that can be assigned the same time slot without interference. TS represents the time slot. Actually, the distance between two nodes can be used to judge whether they will incur transmission interference. For example, two nodes can transmit in the same time slot without interference if their distance is longer than double the largest transmission radius. Also, a more elaborate approach like Zone() in Formulation I can be used to avoid transmission interference.

Due to the algorithm Imp2AppMLST $(G)$, Algorithm I can greatly minimize the energy cost. However, the delay may not be minimized enough because MLST may not be very symmetrical and hence can not reuse time slots efficiently. The running time of the process of assigning time slots is $\Theta\left(n^{2}\right)$, so Algorithm I is near linear with a running time of $\Theta\left(2 n^{2}+m\right)$.

\subsection{Algorithm II for MEMD Multicast}

According to the analysis in the section above, as Algorithm I considers the energy cost and the delay respectively, the latter object may not be optimized enough. To improve delay result, we offer a new approach in Algorithm II (see Fig. 3) for MEMD multicast, which assigns time slots in the generation process of the approximate MLST of the network graph.

\section{Algorithm II $G=(V, E, r)$}

1. $E S:=\{r\}$

2. $T S:=0$;

3. while $E S \neq \varnothing$ do

4. $S:=\varnothing$;

5. Select a vertex $v$ from $E S$ with the largest remained degree;

6. $S:=S \cup\{v\}$;

7. if $v$ 's remained degree $>1$ then $t d:=1$; else $t d:=0$;

8. Put other vertices of $E S$ in $S$ randomly as many as possible satisfying: their remained degree is larger than $t d$ and they can be assigned the same time slot with the vertices of $S$ without transmission interference;

9. Assign each vertex of $S$ time slot $T S+1$;

10. TS++;

11. Expand the vertices of $S$ and expand their unexpanded neighbors as leaves;

12. Update all vertices' remained degree information;

13. Update $E S$ : put the children of each vertex of $S$ in $E S$;

14. end while;

Fig. 3. Algorithm II for MEMD multicast 
In Algorithm II, ES denotes the set of expanding vertices. Other symbols remain the same meanings in Algorithm I. Line 5 ensures that the current tree can be expanded even if all leaves' remained degree are only 1, so Algorithm II can expand all vertices and get a spanning tree of $G$. Lines 7 and 8 are intent on generating a subset $S$ of $E S$ with vertices as many as possible satisfying that all the vertices of $S$ can be assigned the same time slot without transmission interference.

Unfortunately, in worst cases when the sensors are sparse, the spanning tree generated by Algorithm II is not a 2-approximation MLST of $G$, because this algorithm can not guarantee that the result tree is leafy. However, in general cases, especially in dense wireless sensor networks, the total number of branch nodes of the tree should be close to the result of Algorithm I. Moreover, this algorithm can obtain a better result on delay because it considers this object more elaborately. Simulation results in the next section confirm this analysis. This algorithm is no more complex than Algorithm I even in worst cases. The running time required by this algorithm is $\Theta\left(n^{2}+m\right)$.

\section{Simulation Results}

We propose a wireless sensor network with 200 sensors distributed randomly in a variable square place. The longest transmission radius is $120 \mathrm{~m}$. A direct communication link between two nodes is established if their distance is shorter than $120 \mathrm{~m}$. The link obstacle rate is set to one percent. Moreover, two nodes can transmit in the same time slot without interference if their distance is longer than $2 * 120 \mathrm{~m}$, with a more precise threshold $\sqrt{3} * 120$ m for the root's neighbors.

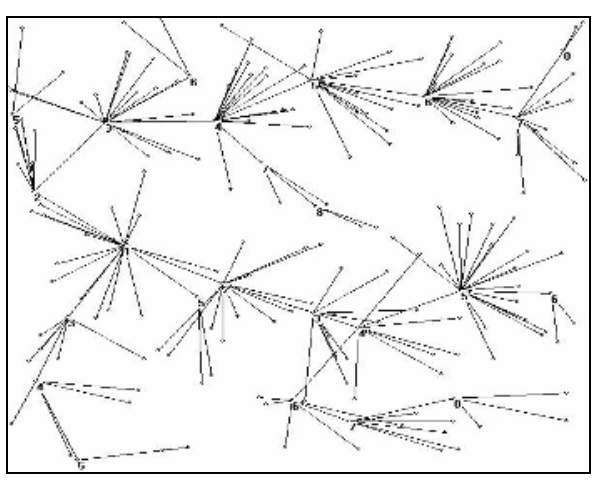

Fig. 4. A sample solution obtained by Algorithm II

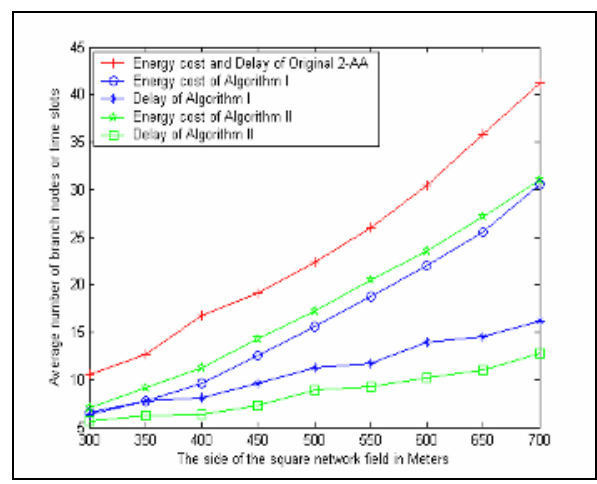

Fig. 5. Simulation results (Original 2-AA denotes the original 2-approximation algorithm for MLST)

A solution example obtained by Algorithm II is shown in Fig. 4. The total number of branch nodes is 24 and only 8 time slots are required. From this example, we have a vivid impression that the energy cost is low and the time slots are reused efficient. Next, the average results will be shown for the algorithms in the sections above. 
By the way, if TDMA is proposed as the access control scheme, each branch node of the multicast tree is assigned a dedicated time slot, and hence both minimum energy cost and minimum delay can be measured by the total number of branch nodes. So the MEMD multicast based on TDMA is also the MLST problem. We implement the original 2-approximation algorithm for the MLST problem here as a solution for this multicast problem. We run the original 2-approximation algorithm, Algorithm I and Algorithm II for networks in different sizes, each of which consists of 1000 random topologies. The average results are shown in Fig. 5.

From the simulation results, we first notice that Algorithm I and Algorithm II work remarkably better than the original 2-approximation algorithm on both energy cost and delay results. Secondly, STDMA outperforms TDMA remarkably, especially when the sensors density is low. Thirdly, these results affirm that our 2-approximation algorithm for MLST, used in Algorithm I, is more efficient than the original one. Moreover, Algorithm I outperforms Algorithm II on energy cost while Algorithm II outperforms Algorithm I on delay. Finally, as mentioned in Section 4.3, the tree obtained by Algorithm II is not a 2-approxiamte MLST in worst cases, however, the average result is close to Algorithm I's and remarkably better than the result of the original 2-approximation algorithm. These results affirm our analysis in the sections above.

\section{Conclusion and Future Work}

In this paper, we have studied the problem of multicast routing with minimum energy cost and minimum delay in wireless sensor networks under STDMA. Firstly, we formulate and explore both MEMD multicast and ME multicast, and show that the latter is just the MLST problem which is NP-complete. Then a more efficient 2-approximation algorithm is proposed for the MLST problem through improving a known one. Based on this algorithm, we obtain a near linear approximation algorithm for the MEMD multicast problem. To further improve the delay result, we also propose another near linear approximation algorithm with a different method for our main problem. Simulation results show that both algorithms have good performance.

In the future, we plan to further study the resource allocation on multicast tree under STDMA and develop a distributed implementation for our multicast schemes. We also plan to consider the multicast problems in mobile wireless sensor networks and Ad Hoc networks.

\section{References}

[1] I. Akyildiz, W. Su, Y. Sankarasubramaniam, and E. Cayirci, "Wireless Sensor Networks: A Survey," Computer Networks, vol. 38, no. 4, March 2002.

[2] J. L. Hill and D. E. Culler, "Mica: a wireless platform for deeply embedded networks", IEEE Micro, vol. 22, no. 6, pp 12 - 24, 2002.

[3] Xiaoxing Guo, "Broadcasting for Network Lifetime Maximization in Wireless Sensor Networks," 2004 First Annual IEEE Communications Society Conference, pp 352 - 358, 2004. 
[4] R. Nelson and L. Kleinrock, "Spatial-TDMA: A collision-free multihop channel access control”, IEEE Transactions on Communications, vol. 33, pp 934 - 944, 1985.

[5] Di.Prieto.R, Mancini.L, "LKHW: a directed diffusion-based secure multicast scheme for wireless sensor networks,.". Proceedings of the 2003 International Conference on Parallel Processing Workshops (ICPPW'03), pp 397 - 406, 2003.

[6] Xiaohua Jia, Deying Li, "Multicast routing with minimum energy cost in ad hoc wireless networks,". GLOBECOM '04, IEEE vol. 5, pp 2897 - 2901, 2004.

[7] Peng-Jun Wan, Calinescu, G, "Minimum-power multicast routing in static ad hoc wireless networks,". IEEE/ACM Transactions on Networking, vol. 12, Issue 3, pp 507 - 514, 2004

[8] C. Intanagonwiwat, R. Govindan, and D. Estrin, "Directed Diffusion: A Scalable and Robust Communicastion," MOBICOM '00, August 2000.

[9] F. Ye, H. Luo, J. Cheng, S. Lu, and L. Zhang, "A Two-Tier Data Dissemination Model for Large-scale Wireless Sensor Networks," ACM International Conference on Mobile Computing and Network (MOBICOM '02), pp 148 - 159, September 2002.

[10] M. R. Garey, D. S. Johnson, "Computers and Intractability: A guide to the theory of NP-completeness,” W. H. Freeman, San Francisco 1979.

[11] G. Galbiati, F. Maffioli, A. Morzenti, "A short note on the approximability of the maximum leaves spanning tree problem," Information Processing Letters 52, pp 45 - 49, 1994.

[12] S. Arora, C. Lund, R. Motwani, M. Sudan, M. Szegedy, "Proof verification and the hardness of approximation problems," Proceedings of the Thirty-third Annual IEEE Symposium on Foundations of Computer Science, pp 14 - 23, 1992.

[13] S. Arora, S. Safra, "Probabilistic checking of proofs: A new characterization of NP," Proceedings of the Thirty-third Annual IEEE Symposium on Foundations of Computer Science, pp $2-13,1992$.

[14] H. Lu, R. Ravi, “A near-linnear time approximation algorithm for maximum leaf spanning tree,” Journal of Algorithms, Vol. 29, No. 1, pp 132 - 141, 1998.

[15] R. Solis-Oba, "2-approximation algorithm for finding a spanning tree with maximum number of leaves," Proceedings on the 6th Annual European Symposium on Algorithms, LNCS 1461, pp 441 - 452, 1998.

[16] P. Bjorklund, P. Varbrand and D. Yuan, "Resource optimization of spatial TDMA in ad hoc radio networks," In Proceedings of the 2003 INFOCOM, San Francisco, CA, April1-3 2003.

[17] J. Gr"onkvist, "Traffic controlled spatial reuse TDMA in multi-hop radio networks," Proceedings of 9th IEEE International Symposium on Personal, Indoor and Mobile Radio Communications, pp 1203 - 1207, 1998.

\section{Appendix: The Original 2-Approximation Algorithm for MLST}

Let $G=(V, E)$ be an undirected connected graph. The algorithm first builds a forest $F$ by using a sequence of expansion rules, to be defined shortly. Then the trees in $F$ are linked together to form a spanning tree $T$. Every tree $T_{i}$ of $F$ is built by first choosing a vertex of degree at least 3 as its root. Then the expansion rules described in Fig. 1 are used to grow the tree. If a leaf $x$ has at least two neighbors not in $T_{i}$ then the rule shown in Fig. 1(b) is used which places all neighbors of $x$ not belonging to $T_{i}$ as its children. On the other hand, if $x$ has only one neighbor $y$ that does not belong to $T_{i}$ and at least two neighbors of $y$ are not in $T_{i}$, then the rule shown in Fig. 1(a) is used. This rule puts $y$ as the only child of $x$ and all the neighbors of $y$ not in $T_{i}$ are made children of $y$. A tree $T_{i}$ is grown until none of its leaves can be expanded. 


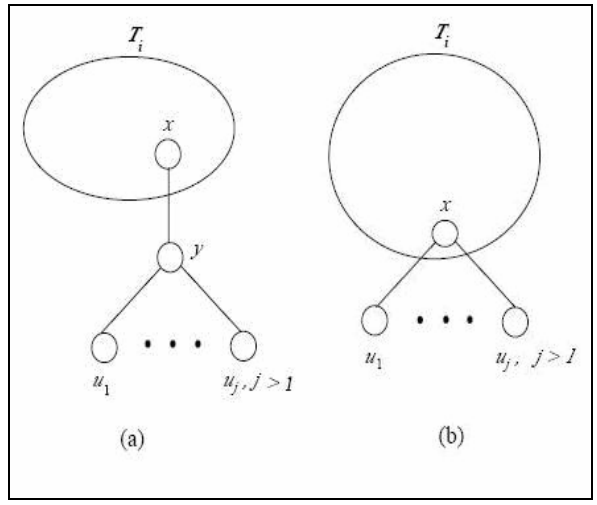

Fig. 1. Expansion rules

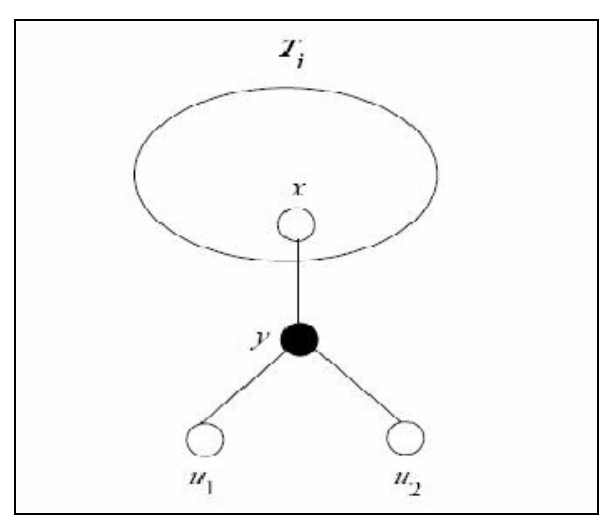

Fig. 2. The rule of priority 1

The expansion rules are assigned priorities as follows. The rule shown in Fig. 2, namely a leaf $x$ has a single neighbor $y$ not in $F$ and $y$ has exactly two neighbors outside $F$, has priority 1 . All other expansion rules have priority 2 . When building a tree $T_{i} \in F$, if two different leaves of $T_{i}$ can be expanded, the leaf that can be expanded with the highest priority rule is expanded first. If two leaves can be expanded with rules of the same priority, then one is arbitrarily chosen for expansion. The full algorithm is shown in Fig. 3.

\section{Algorithm tree $(G)$}

1. $F:=\varnothing$;

2. while there is a vertex $v$ of degree at least 3 do

3. $\quad$ Build a tree $T_{i}$ with root $v$ and expend its neighbors as leaves;

4. while at least one leaf of $T_{i}$ can be expanded do

5. Find a leaf of $T_{i}$ that can be expanded with a rule of largest priority, and expand it;

6. end while;

7. $F:=F \cup T_{i}$;

8. Remove from $G$ all vertices in $T_{i}$ and all edges incident to them;

9. end while

10. Connect the trees in $F$ and all vertices not in $F$ to form a spanning tree $T$;

Fig. 3. The original 2-approxiamtion algorithm for MLST 